\title{
Antibiotikahaltiger Zement: mehr Halt für Hüft-Endoprothesen?
}

\section{Hier steht eine Anzeige.}

Zementierte Hüftgelenke mit Antibiotika im Fixierungsmaterial machen seltener eine Revision notwendig als unzementierte Prothesen, wie die Ergebnisse einer französischen bevölkerungsgestützten Studie mit mehr als 100.000 Patienten vermuten lassen.

E s wird geschätzt, dass nach einem totalen HüftgelenkerE satz (TEP) jährlich bei etwa $1 \%$ der Patienten eine Revisionsoperation erforderlich ist. Um den Einfluss der Fixationstechnik und der Oberflächenbeschaffenheit des Gelenks auf die Revisionshäufigkeit zu prüfen, analysierten französische Orthopäden und Epidemiologen um Dr. Sandrine Colas von der dem französischen Gesundheitsministerium unterstellten Aufsichtsbehörde ANSM (Agence nationale de sécurité du médicament et des produits de santé) Daten des Versicherungssystems in Frankreich. Sie stammten von insgesamt fast 100.200 Patienten, bei denen zwischen April 2010 und Dezember 2011 eine Hüftarthroplastik vorgenommen worden war.

$74,8 \%$ der Patienten erhielten ein unzementiertes Implantat, $3,8 \%$ eine TEP mit antibiotikafreier und 21,4 \% eine mit antibiotikahaltiger Zementierung. Die Autoren hatten auch die Gelenkoberfläche im Blick: Sie verglichen die Revisionsraten bei Keramik-auf-Keramik- (40,9\%), Keramik-auf-Polyethylen$(20,8 \%)$ und Metall-auf-Metall-Implantaten $(4,4 \%)$ mit jener bei Metall-auf-Polyethylen-Prothesen (33,9\%). Primärer Endpunkt der Studie war die Rate an Revisionen, bei denen das Implantat ausgetauscht oder entfernt wurde.

Die mit antibiotikahaltigem Zement verankerten Prothesen hatten insgesamt eine signifikant bessere Prognose als die nicht zementierten Kunstgelenke. Die kumulative Revisionsrate lag bei 2,4 versus 3,3\% ( $p<0,001)$. Beim Vergleich der Revisionsraten zwischen Patienten mit Keramik-auf-Polyethylen- und Keramik-auf-Keramik-Prothesen und Patienten mit Metall-auf-Polyethylen-Gelenken ergaben sich keine signifikanten Unterschiede.

Fazit: Das Risiko für eine Revision war bei Verwendung antibiotikahaltigen Zements um $26 \%$ niedriger als in der Vergleichsgruppe (Hazard Ratio: 0,74; 95\%-Konfidenzintervall [KI] 0,67-0,84; $\mathrm{p}<0,001)$. Nach multivariater Analyse betrug der Wert 0,77 (95\%-KI 0,70-0,85). Als Nachteil der Studie nannte die Kommentatorin Dr. Constance R. Chu von der Stanford-Universität in Redwood City unter anderem das kurze Follow-up von weniger als drei Jahren. Um eine relevante Aussage machen zu können, sei ein Follow-up von mindestens zehn Jahren erforderlich.

Peter Leiner

Colas S et al. Association Between Total Hip Replacement Characteristics and 3-Year Prosthetic Survivorship: A Population-Based Study. JAMA

Surg 2015; online 19. August; doi: 10.1001/jamasurg.2015.1325 